Disponible en ligne : www.cahiersagricultures.fr

\title{
Les oasis en Afrique du Nord: dynamiques territoriales et durabilité des systèmes de production agricole
}

\author{
Ahmed Bouaziz $^{1}$, Ali Hammani ${ }^{1}$ et Marcel Kuper ${ }^{1,2, *}$ \\ ${ }^{1}$ Institut Agronomique et Vétérinaire Hassan II, Médinat El Irfane, Rabat, Maroc \\ ${ }^{2}$ Université de Montpellier, Cirad, Umr G-Eau, Montpellier, France
}

\begin{abstract}
Résumé - Les oasis sahariennes ont connu des changements particulièrement profonds et déstabilisants dans la seconde moitié du $20^{\mathrm{e}}$ siècle, s'accompagnant de transformations sociales, économiques, agricoles et environnementales. Aux oasis phoenicicoles étagées classiques, viennent s'adjoindre de nouvelles extensions ou projets agricoles à travers des initiatives étatiques ou locales. Dans un contexte où les formes d'agriculture les plus modernes et les plus traditionnelles sont juxtaposées, il est pertinent d'analyser à la fois les ruptures et les continuités entre ces différentes formes d'agriculture saharienne. C'est précisément dans le but de contribuer à améliorer et partager les connaissances sur les dynamiques agricoles et territoriales en cours dans les oasis en Afrique du Nord, que nous avons organisé un symposium international à Zagora, oasis de peuplement millénaire. Le présent numéro thématique prolonge cette discussion sur ces dynamiques au sein des anciennes et nouvelles oasis et leurs interrelations avec les modes d'élevage et les activités non agricoles. Les différentes contributions au numéro thématique montrent les risques induits par les changements rapides. Elles dévoilent aussi des oasis bien vivantes, dont la durabilité passe sans doute par la cohabitation d'initiatives diversifiées.
\end{abstract}

Mots clés : oasis / Maghreb / eau souterraine / agriculture / palmier dattier

Abstract - Oases in North Africa: territorial dynamics and sustainability of farming systems. The Saharan oases experienced particularly profound and destabilizing changes in the second half of the 20th century, accompanied by social, economic, agricultural and environmental transformations. New extensions and agricultural projects have been implemented through a multitude of state or local initiatives. In a context where the most modern and traditional forms of agriculture are juxtaposed, it is important to analyze not only the ruptures, but also the continuities between these different forms of Saharan agriculture. It is precisely with the aim of improving and sharing knowledge on the agricultural and territorial dynamics underway in the oases in North Africa, that we organized an international symposium in Zagora, a millennial settlement oasis. This thematic issue continues the discussion of these dynamics within the old and new oases and their interrelations with farming and non-agricultural activities. The various contributions to the thematic issue show the risks of rapid change. They also reveal living oases, whose sustainability will undoubtedly depend on the coexistence of diversified initiatives.

Keywords: oasis / North Africa / groundwater / agriculture / date palm

\section{Introduction: un regard dynamique sur les différentes formes d'agricultures sahariennes}

L'oasis (littéralement «lieu habité ») est un espace cultivé intensivement dans un milieu désertique. Ces écosystèmes domestiqués, fondés sur une multitude de méthodes de

\footnotetext{
*Auteur de correspondance : marcel.kuper@cirad.fr
}

mobilisation de l'eau, partagent un certain nombre de caractéristiques tout à fait originales: des systèmes de polyculture-élevage, un étagement de la végétation dont la strate dominante est souvent constituée de palmiers dattiers, des systèmes de cultures très intensifs, et une organisation collective de l'espace (Toutain, 1987; Battesti, 2005). Ce trait commun recouvre cependant une grande diversité de formes dans les oasis d'Afrique du Nord, que l'on peut distinguer selon leur économie (oasis relais versus oasis de peuplement, par exemple, ou encore les nouvelles oasis «modernes» liées 
au développement de l'accès motorisé aux eaux souterraines), leur situation agro-climatique (oasis côtières, oasis d'altitude, oasis continentales), ou leur structure, en particulier selon les systèmes hydrauliques permettant de mobiliser et distribuer l'eau d'irrigation (Toutain, 1987; Battesti, 2005).

Longtemps considérées en déclin, les oasis sahariennes ont connu un « renouveau » étonnant dans la seconde moitié du $20^{\mathrm{e}}$ siècle: «l'agriculture saharienne... a conquis des terres nouvelles, a renouvelé ses méthodes, et se présente comme un vaste front pionnier, multiformes et multilieux » (Côte, 2002: 6). Selon l'endroit, il s'agit d'une agriculture d'entrepreneurs (le cas Libyen avec ses pivots céréaliers est assez emblématique) ou au contraire d'une agriculture reposant sur de solides paysanneries, tout en mobilisant les eaux souterraines à travers des forages profonds, par exemple dans les Ziban en Algérie ou le Nefzaoua en Tunisie (Côte, 2002). Aux oasis phoenicicoles classiques, viennent «s'adjoindre des emprises agricoles» que l'on qualifie de «projets agricoles» ou plus récemment de "périmètres de mise en valeur » ou «d'attributions foncières » (Bisson, 2003 : 193), ou encore de projets d'investissement, ou d'extensions. Dans une telle situation, où les formes d'agriculture les plus modernes et les plus traditionnelles sont juxtaposées (Côte, 2002), il est pertinent d'analyser à la fois les ruptures et les continuités entre ces différentes formes (Lavie et Marshall, 2017). Il s'agit, d'une part, de dépasser le regard nostalgique (et souvent orientaliste) sur l'oasis traditionnelle qui devrait être préservée et mise sous cloche, et d'autre part, de nuancer, sans doute, le constat d'échec des formes modernes d'agricultures sahariennes $\mathrm{du}$ fait de leur inadaptation constatée à un contexte oasien. Il n'y a pas seulement une certaine continuité territoriale ou même des interdépendances entre ces différentes formes, mais aussi, dans les deux cas, des mutations agricoles et territoriales qui peuvent être observées par un regard dynamique sur ces agrosystèmes. En effet, dans un tel contexte hostile, cet écosystème «hyper-anthropisé» peut être vu «comme un équilibre instable, un équilibre qui doit, à tout moment, être ajusté par l'homme sous forme de travail» (Battesti, 2005: 16).

En 1987, Toutain (1987: 242) soulignait l'importance pour la recherche agronomique d'avoir une connaissance fine des dynamiques agricoles en régions sahariennes afin d'accompagner les projets de développement:«Il y a quelque 25 ans, quand il apparut nécessaire politiquement de mettre en valeur les oasis, soit par des actions de rénovation ou de création, la recherche, en général, et, en particulier, agronomique, qui a pour rôle d'appuyer scientifiquement et techniquement les opérations de développement n'était pas armée pour participer à l'élaboration des projets. Ainsi, s'est-elle montrée incapable de proposer et de justifier des alternatives aux projets de développement importés et basés sur l'injection de paquets de technologies modernes qui se sont montrées très vite inadaptées et, souvent, néfastes en régions sahariennes. »

C'est précisément dans le but de contribuer à améliorer et partager les connaissances sur les dynamiques agricoles et territoriales en cours dans les régions sahariennes qu'il nous a été demandé par l'Agence nationale pour le développement des zones oasiennes et de l'arganier (ANDZOA) du Maroc, d'organiser un symposium international sur «Les dynamiques territoriales et la durabilité des systèmes de production agricole dans les zones oasiennes en Afrique du Nord». Ce colloque a réuni du 13 au 15 avril 2016 à Zagora, oasis de peuplement millénaire, plus d'une centaine de personnes des mondes de la recherche et du développement, ainsi que des ONG.

Quatre thèmes ont été retenus pour l'atelier, dont les communications ont été documentées dans les actes du symposium (Bouaziz et al., 2017) :

- systèmes de production oasiens (17 communications);

- valorisation et marketing des produits oasiens $(8 \mathrm{commu}-$ nications);

- développement et dynamiques des territoires sahariens (12 communications);

- gestion durable des ressources naturelles dans les oasis (14 communications).

Le présent numéro thématique a pour objectif de prolonger cette discussion sur les évolutions et leurs conséquences sur l'environnement et l'utilisation des ressources naturelles, au sein des anciennes et nouvelles oasis et leurs interrelations avec les modes d'élevage et les activités non agricoles (tourisme, artisanat, mines...). Nous avons cherché à analyser ces évolutions à travers la diversité des situations qu'on y rencontre.

\section{Les contributions au numéro thématique}

Le numéro thématique commence par une mise en perspective des oasis sahariennes à travers un voyage qui amène le lecteur dans les Amériques arides, l'Extrême-Orient chinois, les métropoles d'Asie centrale - telles que Samarkand ou Tachkent-pour «revenir aux oasis recluses du grand Sahara africain » (Fassi, 2017). Le propos de Fassi, actif dans le comité marocain «Man \& Biosphere» de l'Unesco, est de considérer l'oasis comme «le modèle fondamental de la durabilité ».

Une série de trois articles porte ensuite sur la physiologie et le développement du palmier dattier. Le premier article s'intéresse à l'efficience d'utilisation de l'eau d'irrigation pour le palmier. Dans le but d'améliorer cette efficience, Sabri et al. (2017) ont conçu et testé des régimes d'irrigation déficitaire pour évaluer leur impact sur la croissance et le développement foliaire du palmier. Ils montrent qu'il est possible, pour le cas du Tafilalet (Maroc), d'économiser jusqu'à $14 \%$ du volume d'eau sans effet négatif sur le développement du palmier. Ce résultat est complété par des travaux publiés ailleurs montrant que ce régime déficitaire n'a pas d'impact non plus sur les rendements en dattes. Le deuxième article propose une méthode de lutte contre la fusariose vasculaire du palmier dattier, le bayoud, à travers une méthode couplant la solarisation et la fumigation (Essarioui et Sedra, 2017). Ce travail, qui prolonge une longue série de travaux sur le bayoud depuis près d'un siècle (Saaidi, 1992), offre des perspectives de traitement localisé de cette maladie, en particulier dans de nouvelles plantations qui ne sont pas (encore) infestées à grande échelle. Le troisième article de cette série s'intéresse à un outil biotechnologique, les champignons mycorhiziens arbusculaires (CMA), pour aider les palmiers à tolérer les conditions difficiles des palmeraies au Maroc, en particulier le déficit hydrique et le bayoud (Meddich et al., 2017). Les auteurs montrent que les CMA autochtones, très adaptés au contexte édaphique local, constituent une voie intéressante 
pour améliorer la croissance des palmiers dattiers face aux contraintes du milieu. Ces trois travaux sont complétés par une étude proposant une méthode, utilisant les données des registres géométriques et radiométriques, pour caractériser la diversité des systèmes de culture dans les oasis, appliquée à la très réputée oasis de Nefta en Tunisie (Fargette et al., 2017). La méthode permet d'abord de détecter les jardins en (quasi) abandon et de distinguer la palmeraie «traditionnelle», dense et étagée, de la palmeraie «moderne» en mono-culture. Elle permet aussi de mettre en lumière ce que les auteurs appellent des jardins mixtes, qui sont souvent de nouvelles plantations de palmier dattier, associées à des cultures annuelles.

Faye et al. (2017) pour le dromadaire, suivi de Sraïri et al. (2017) pour l'ensemble du cheptel dans toute sa diversité (ovins, caprins, bovins, camelins), rappellent le rôle important de l'élevage dans les systèmes agraires oasiens. Les deux articles actualisent les connaissances de l'élevage dans ces systèmes et dressent un tableau de fortes mutations. Ainsi, Faye et al. (2017) ont analysé le développement d'un élevage camelin périurbain (lait, viande) qui «s'investit dans une certaine modernité dont l'oasis et ses habitants ne peuvent que tirer profit pour leur développement». Sraïri et al. (2017) soulignent, pour les oasis de la vallée du Drâa (Maroc), un début de spécialisation de l'élevage, en particulier l'intensification de la production ovine, centrée autour de la sélection de la race D'man, et l'intensification de l'élevage bovin. Ces deux stratégies se révèlent cependant assez risquées et peu performantes pour le moment. Les auteurs plaident, en conséquence, pour une prise en compte de la diversité des types d'élevages afin de mieux valoriser les ressources locales, $\mathrm{y}$ compris pastorales, dans un milieu naturel fortement contraint.

Les oasis bénéficient d'une bonne image de marque que plusieurs initiatives étatiques et locales souhaitent mettre en valeur. Carpentier (2017) analyse les multiples initiatives, à l'échelle individuelle et collective, pour la valorisation du territoire oasien (élevage, tourisme local, spécialisation des cultures, démarche de qualité, etc.). Ces stratégies sont foisonnantes et parfois contradictoires (spécialisation, intensification ou diversification?), mais l'auteure montre que la durabilité des systèmes oasiens passe par «la cohabitation d'initiatives diversifiées», chaque forme de valorisation apportant « une réponse socialement différenciée aux contraintes de l'oasis, entre promotion d'une petite agriculture familiale diversifiée, consolidation d'exploitations dominantes, et maintien d'exploitations de taille moyenne». Harrak et al. (2018) et Benziouche (2017) poursuivent cette réflexion, en focalisant sur quelques-unes de ces initiatives. Harrak et al. (2018) s'intéressent au savoir-faire traditionnel des oasiennes pour la préparation des pâtes de dattes dans les oasis de Tata (Maroc). Les auteurs proposent un procédé amélioré pour la préparation et font des recommandations pratiques pour le conditionnement et le stockage des pâtes. Benziouche (2017) analyse le système de production de dattes biologiques dans les Ziban (Algérie). L'auteur relève les différentes opportunités présentes (système de production non mécanisé, faible utilisation des intrants chimiques, présence de variétés précoces et tardives, savoir-faire local, appui politique) qui pourraient favoriser une conversion relativement aisée vers l'agriculture biologique. Cependant, ces opportunités sont encore faiblement exploitées et les producteurs sont focalisés sur l'accroissement des volumes de production, la demande pour les dattes conventionnelles étant en croissance continue.

Bien que les oasis aient été très tôt intégrées à l'économie de marché, contrairement à un certain mythe d'insularité, les changements endogènes (émigration, urbanisation...) et exogènes (projets étatiques, arrivée d'investisseurs attirés par le potentiel économique offert) sont particulièrement profonds et déstabilisants depuis la seconde moitié du $20^{\mathrm{e}}$ siècle (Lavie et Marshall, 2017). La cohabitation de différentes formes d'agriculture, de la palmeraie classique étagée jusqu'aux plantations phoenicicoles en mono-culture ou encore les champs de blé sous pivot importé, interpelle les différents acteurs de la recherche et du développement depuis longtemps, selon différentes perspectives: les résultats mitigés de la « grande » mise en valeur, en particulier céréalière (Otmane et Kouzmine, 2013), l'exploitation intensive des eaux souterraines profondes et leurs impacts environnementaux et socioéconomiques (mise en péril des systèmes hydrauliques à foggara, par exemple; Remini et al., 2011), ou encore la permanence et l'adaptation des paysanneries (Bisson, 1992; Bendjelid et al., 1999). Trois articles dans ce numéro thématique contribuent à ce débat «tradition-modernité». Hadeid et al. (2018) suggèrent qu'en Algérie de nouvelles dynamiques agricoles sont perceptibles dans l'agriculture saharienne, qu'elle soit traditionnelle ou moderne : « la période actuelle est ainsi caractérisée non par une concurrence de l'agriculture moderne envers l'agriculture oasienne mais vers le développement d'une autre vision fondée sur le profit à caractère libéral». Les différentes formes d'agriculture montreraient donc «une forme d'adaptation au contexte politique et économique du pays ». Idda et al. (2017) focalisent leur analyse sur le système hydraulique à foggara dans le Touat (Algérie), montrant comment les oasiens ont réussi à construire de nouveaux dispositifs hydrauliques hybrides en alimentant les foggaras par des forages. Les auteurs montrent surtout que ce changement technique est accompagné par des transformations sociales, économiques et environnementales, montrant des oasis bien vivantes. Ould Rebaï et al. (2017) analysent la conception et la diffusion d'un petit pivot artisanal à Oued Souf (Algérie), qui est utilisé en particulier pour irriguer la pomme de terre à partir du pompage des eaux souterraines. Les auteurs montrent que le pivot artisanal résulte d'une innovation incrémentielle à partir des grands pivots conventionnels introduits par l'État, qui étaient coûteux et non adaptés aux objectifs et aux capacités techniques des agriculteurs.

Le «déverrouillage» des eaux souterraines profondes dans l'espace saharien, basé sur un accès privé à l'eau par des forages profonds et visant une agriculture de marché, a profondément changé les rapports à l'eau dans un milieu où la ressource était traditionnellement vue comme rare (Hamamouche etal., 2015). Certains observateurs qualifient cette exploitation de l'eau souterraine dans le Sahara de minière, entraînant des baisses régulières des nappes (Margat, 2008); ils constatent des pratiques de sur-irrigation rendues possibles grâce à des eaux «modernes» d'accès et de gestion plus faciles, conduisant à une «infamie», c'est-à-dire des oasis malades de trop d'eau (Côte, 1998). Marc Côte plaide donc pour appréhender la gestion de l'eau dans sa globalité : irriguer, drainer, pomper, évacuer. Trois contributions alimentent ce débat sur la gestion de l'eau, sous différents angles. Tout d'abord, Daoudi et al. (2017) montrent la complémentarité 
existant entre le cadre formel (les règlements officiels) et les pratiques locales (informelles mais tolérées) d'accès à l'eau, qui explique la dynamique agricole actuelle du Sahara algérien. L'État «tolérant» n'est pas absent de cette dynamique et pourrait s'il en avait la volonté politique " reprendre la main pour réguler l'usage à l'échelle régionale ». El Khoumsi et al. (2017) montrent l'importance de la nappe phréatique dans le Tafilalet (Maroc), non seulement comme source d'approvisionnement des agricultures oasiennes à travers le pompage, mais aussi pour alimenter directement des palmiers dattiers par remontée capillaire. Les auteurs montrent que jusqu'à $50 \%$ de la transpiration peut être ainsi fournie. Enfin, Bourziza et al. (2017) proposent de revenir à des modes d'irrigation qui reconnaissent la rareté de l'eau. Le goutte à goutte enterré s'avère intéressant et économe comme mode d'irrigation. De plus, ce mode d'irrigation peut se conjuguer avec d'autres sources d'approvisionnement, comme l'eau de crue (importante pour le lessivage des sels et l'amélioration de la qualité de la nappe phréatique) et la remontée capillaire de la nappe phréatique.

\section{Conclusion}

Le débat sur les dynamiques agricoles dans les oasis sahariennes, auquel ce numéro thématique ambitionne de contribuer n'est, à l'image de ces mêmes dynamiques, jamais fini: «L'équilibre d'une société vis-à-vis de son milieu n'existe jamais en tant que tel, il est toujours remis en cause par l'apparition de nouveaux événements ou phénomènes historiques, naturels ou sociaux. Peut-être ne faut-il pas lire trop rapidement un changement dans les relations au milieu comme une perte d'harmonie, mais comme l'histoire du procès incessant de la poursuite (recherche) des équilibres ") (Battesti, 2005 : 30). Nous espérons donc que le symposium de Zagora et ce numéro thématique qui en est le prolongement, encourageront de nouvelles recherches, pluridisciplinaires, sur les oasis sahariennes et leur durabilité. Les différentes contributions montrent que les oasis sont à la fois très spécifiques du fait des fortes contraintes climatiques, mais aussi très intégrées dans les grandes évolutions contemporaines: opposition tradition/modernité, évolution du rôle de l'État, des communautés locales, et des acteurs privés, reconnaissance de spécificités de terroirs, souci de la durabilité des ressources naturelles, préoccupations environnementales. C'est ainsi, comme le propose Fassi (2017), que la zone oasienne se trouve aux avant-postes d'une réflexion plus universelle sur la transition écologique vers le développement durable.

Remerciements. Les auteurs de cet éditorial tiennent à remercier toutes les personnes et institutions qui ont contribué à la réussite du symposium de Zagora et de ce numéro thématique sur les oasis, notamment, l'ANDZOA, l'Inra Maroc, l'IAV Hassan II, ainsi que la KIADPAI (Khalifa International Award for Date Palm and Agricultural Innovation, UAE), la DRA (Direction régionale de l'agriculture) de Draa-Tafilalet, les ORMVA (Offices régionaux de mise en valeur agricole) de Tafilalet et de Ouarzazate, ainsi que MM. le Gouverneur et le Secrétaire général de la province de Zagora.

\section{Références}

Battesti V. 2005. Jardins au désert, Évolution des pratiques et savoirs oasiens, Jérid tunisien. Paris: IRD, Coll. À travers champs.

Bendjelid A, Dari O, Hadeid M, Bellal SA, Gacem F, Belmahi MN, et al. 1999. Mutations sociales et adaptation d'une paysannerie ksourienne du Touat: Ouled Hadj Mamoun (wilaya d'Adrar, Algérie). Insaniyat/إنسانيات. Revue Algérienne d'Anthropologie et de Sciences Sociales (7): 39-52.

Benziouche S. 2017. L'agriculture biologique, un outil de développement de la filière dattes dans la région des Ziban en Algérie. Cah Agric 26(3): 35008. DOI: 10.1051/cagri/2017025.

Bisson J. 1992. Les foggaras du Sahara algérien : déclin ou renouveau. In: Balland D, ed. Les eaux cachées. Études géographiques sur les galeries drainantes souterraines. Paris (France): Univ. Paris Sorbonne, pp. 7-26.

Bisson J. 2003. Mythes et réalités d'un désert convoité : le Sahara. Paris : L'Harmattan.

Bouaziz A, Hammani A, Kuper M. 2017. Gestion intégrée et durable des territoires oasiens. Actes du colloque international des Oasis, Zagora, 13-15 avril 2016. Rabat: ANDZOA, IAV Hassan II, INRA.

Bourziza R, Hammani A, Mailhol JC, Bouaziz A, Kuper M. 2017. Modélisation de l'irrigation en goutte à goutte enterré du palmier dattier sous les conditions oasiennes. Cah Agric 26(3): 35007. DOI: $10.1051 /$ cagri/2017023.

Carpentier I. 2017. Diversité des dynamiques locales dans les oasis du Sud de la Tunisie. Cah Agric 26(3): 35001. DOI: 10.1051/cagri/ 2017017.

Côte M. 1998. Des oasis malades de trop d'eau? Science et changements planétaires/Sécheresse 9(2): 123-130.

Côte M. 2002. Des oasis aux zones de mise en valeur: l'étonnant renouveau de l'agriculture saharienne. Méditerranée 99(3): 5-14.

Daoudi A, Lejars C, Benouniche N. 2017. La gouvernance de l'eau souterraine dans le Sahara algérien : enjeux, cadre légal et pratiques locales. Cah Agric 26(3): 35004. DOI: 10.1051/cagri/2017021.

El Khoumsi W, Hammani A, Bouarfa S, Bouaziz A, Ben Aïssa I. 2017. Contribution de la nappe phréatique à l'alimentation hydrique du palmier dattier (Phoenix dactylifera) dans les zones oasiennes. Cah Agric 26(4): 45005. DOI: 10.1051/cagri/2017032.

Essarioui A, Sedra MH. 2017. Lutte contre la maladie du bayoud par solarisation et fumigation du sol. Une expérimentation dans les palmeraies du Maroc. Cah Agric 26(4): 45010. DOI: 10.1051/ cagri/2017043.

Fargette M, Loireau M, Raouani N, Kadri ZM, Simon H, Libourel T, et al. 2017. Modélisation de la diversité des systèmes de culture, de l'observation à la détection : cas de l'oasis de Nefta (Djérid, Tunisie). Cah Agric 26(4): 45009. DOI: 10.1051/cagri/2017042.

Fassi D. 2017. Les oasis du Monde, carrefour des civilisations et modèle fondamental de durabilité. Cah Agric 26(4): 46001. DOI: $10.1051 /$ cagri/2017037.

Faye B, Senoussi H, Jaouad M. 2017. Le dromadaire et l'oasis : du caravansérail à l'élevage périurbain. Cah Agric 26(1): 14001. DOI: $10.1051 /$ cagri/2017005.

Hadeid M, Bellal SA, Ghodbani T, Dari O. 2018. L'agriculture au Sahara du sud-ouest algérien: entre développement agricole moderne et permanences de l'agriculture oasienne traditionnelle. Cah Agric 27(1). DOI: 10.1051/cagri/2017060.

Hamamouche MF, Kuper M, Lejars C. 2015. Émancipation des jeunes des oasis du Sahara algérien par le déverrouillage de l'accès à la terre et à l'eau. Cah Agric 24(6): 412-419. DOI: 10.1684/ agr.2015.0777. 
Harrak H, Hamouda A, Nadi M. 2018. Évaluation et amélioration de la qualité des pâtes traditionnelles de dattes, produits du terroir des oasis. Cah Agric 27(1): 15001. DOI: 0.1051/cagri/2017057.

Idda S, Bonté B, Mansour H, Bellal SA, Kuper M. 2017. Monument historique ou système bien vivant? Les foggaras des oasis du Touat (Algérie) et leur réalimentation en eau par pompage. Cah Agric 26(5): 55007. DOI: $10.1051 /$ cagri/2017049.

Lavie E, Marshall A. (Eds.). 2017. Oases and globalization: ruptures and continuities. Cham (Switzerland): Springer.

Margat J. 2008. Exploitations et utilisations des eaux souterraines dans le monde. Paris : Unesco/BRGM. http://www.hydrologie.org/ BIB/Publ UNESCO/SOG 1598 70A.pdf.

Meddich A, Ait El Mokhtar M, Wahbi S, Boumezzough A. 2017. Évaluation des potentialités mycorhizogènes en lien avec les paramètres physico-chimiques des sols de palmeraies du Maroc (Marrakech et Tafilalet). Cah Agric 26(4): 45012. DOI: 10.1051/ cagri/2017044.

Otmane T, Kouzmine Y. 2013. Bilan spatialisé de la mise en valeur agricole au Sahara algérien. Mythes, réalisations et impacts dans le Touat-Gourara-Tidikelt. Cybergeo: European Journal of Geography 632: 1-27. DOI: 10.4000/cybergeo.25732.
Ould Rebaï A, Hartani T, Chabaca MN, Kuper M. 2017. Une innovation incrémentielle : la conception et la diffusion d'un pivot d'irrigation artisanal dans le Souf (Sahara algérien). Cah Agric 26(3): 35005. DOI: 10.1051/cagri/2017024.

Remini B, Achour B, Albergel J. 2011. Timimoun's foggara (Algeria): an heritage in danger. Arabian Journal of Geosciences 4(3-4): 495-506.

Saaidi M. 1992. Comportement au champ de 32 cultivars de palmier dattier vis-à-vis du bayoud : 25 années d'observations. Agronomie 12(5): 359-370.

Sabri A, Bouaziz A, Hammani A, Kuper M, Douaik A, Badraoui M. 2017. Effet de l'irrigation déficitaire contrôlée sur la croissance et le développement foliaire du palmier dattier (Phoenix dactylifera L.). Cah Agric 26(5): 55005. DOI: 10.1051/cagri/2017033.

Sraïri MT, M'ghar FA, Benidir M, Bengoumi M. 2017. Analyse typologique de la diversité et des performances de l'élevage oasien. Cah Agric 26(1): 15005. DOI: 10.1051/cagri/2017002.

Toutain G. 1987. Approche globale d'un milieu oasien et préhension des problèmes de mise en valeur agricole (Sud Marocain). In : Antheaume B, Blanc Pamard C, Diallo Y, Lassailly JV, eds. Le développement rural: comprendre pour agir. Paris: ORSTOM, pp. 239-307.

Citation de l'article : Bouaziz A, Hammani A, Kuper M. 2018. Les oasis en Afrique du Nord: dynamiques territoriales et durabilité des systèmes de production agricole. Cah. Agric. 27: 14001. 